International Journal of Mathematical Analysis

Vol. 9, 2015, no. 3, 111 - 116

HIKARI Ltd, www.m-hikari.com

http://dx.doi.org/10.12988/ijma.2015.411353

\title{
Boundary Value Problem for Second Order Ordinary Linear Differential Equations with Variable Coefficients
}

\author{
A. Tungatarov \\ Department of Mechanics and Mathematics \\ Al-Farabi Kazakh National University \\ av. al-Farabi 71, 050012 Almaty, Kazakhstan \\ D. K. Akhmed-Zaki \\ Department of Mechanics and Mathematics \\ Al-Farabi Kazakh National University \\ av. al-Farabi 71, 050012 Almaty, Kazakhstan
}

Copyright (c) 2014 A. Tungatarov and D. K. Akhmed-Zaki. This is an open access article distributed under the Creative Commons Attribution License, which permits unrestricted use, distribution, and reproduction in any medium, provided the original work is properly cited.

\begin{abstract}
In this article the general solution of one class of second order ordinary differential equations with variable coefficients is fined. The twopoint boundary value problem for this class is solved.
\end{abstract}

\section{Mathematics Subject Classification: 34C30}

Keywords: Second order, ordinary differential equation, two-point boundary value problem, variable coefficients

\section{Introduction}

Let $0<x_{1}<\infty, S\left[0, x_{1}\right]$ is the class of measurable, essentially bounded functions $f(x)$ in $\left[0, x_{1}\right]$ and $W_{\infty}^{2}\left[0, x_{1}\right]$ is the class of functions $f(x)$, for which 
$\frac{d^{2} f}{d x^{2}} \in S\left[0, x_{1}\right]$. The norm of an element from $S\left[0, x_{1}\right]$ is defined by the formula

$$
|f|_{0}=\operatorname{essup}_{x \in\left[0, x_{1}\right]}|f(x)|=\lim _{p \rightarrow \infty}\|f\|_{L_{p}\left[0, x_{1}\right]} .
$$

We consider the equation

$$
\frac{d^{2} u}{d x^{2}}+a(x) u=f(x)
$$

in interval $\left[0, x_{1}\right]$, where $a(x), f(x) \in S\left[0, x_{1}\right]$.

Two-point boundary value problems for second order ordinary differential equations are classical area of research of the theory of ordinary differential equations and because of their broad application in mechanic, mathematical physic and geometry (see, for example,[1]-[9]) they are still actively investigated. However, in mathematical literature the equations of the form (1) with continuous coefficients are studied and sufficient conditions of resolvability of boundary value problems for them are received. In author's works $[10,11]$ the general solution of equation (1) is constructed and Cauchy problem for it with initial point $x=0$ is solved. In this work an explicit form of general solution of equation (1) in class

$$
W_{\infty}^{2}\left[0, x_{1}\right] \bigcap C^{1}\left[0, x_{1}\right]
$$

where

$$
x_{1}<\sqrt{\frac{2}{|a|_{0}}}
$$

is found and next two - point boundary value problem is solved.

Problem D. Find the solution of equation (1) from the class (2) satisfying the conditions

$$
u(0)=\alpha, \quad u^{\prime}\left(x_{1}\right)=\beta,
$$

where $\alpha, \beta$ are given real numbers.

\section{Construction of the general solutions to equa- tion (1)}

By integrating two times the equation (1), we get

$$
u(x)=(B u)(x)+g(x)+c_{1} x+c_{2},
$$

where $c_{1}, c_{2}$ are any real numbers, 


$$
(B u)(x)=\int_{0}^{x} \int_{y}^{x_{1}} a(t) u(t) d t d y, \quad g(x)=\int_{0}^{x} \int_{y}^{x_{1}} f(t) d t d y .
$$

Applying the operator $B$ to both sides of equation (5) we have

$$
(B u)(x)=\left(B^{2} u\right)(x)+(B g)(x)+c_{1} a_{1}(x)+c_{2} b_{1}(x),
$$

where

$$
\begin{gathered}
\left(B^{2} u\right)(x)=(B(B u)(x))(x), \\
a_{1}(x)=\int_{0}^{x} \int_{y}^{x_{1}} t a(t) d t d y, \quad b_{1}(x)=\int_{0}^{x} \int_{y}^{x_{1}} a(t) d t d y .
\end{gathered}
$$

From (5) and (6)it follows

$$
u(x)=\left(B^{2} u\right)(x)+c_{1}\left(x+a_{1}(x)\right)+c_{2}\left(1+b_{1}(x)\right)+g(x)+(B g)(x) .
$$

Further we use following functions and operators:

$$
\begin{gathered}
a_{k}(x)=\int_{0}^{x} \int_{y}^{x_{1}} a(t) a_{k-1}(t) d t d y, \quad b_{k}(x)=\int_{0}^{x} \int_{y}^{x_{1}} a(t) b_{k-1}(t) d t d y, \\
\left(B^{k} u\right)(x)=\left(B\left(B^{k-1} u\right)(x)\right)(x), \quad(k=2,3, \ldots) .
\end{gathered}
$$

Applying the operator $B$ to both sides of equation (7) we get

$$
\begin{gathered}
(B u)(x)=\left(B^{3} u\right)(x)+c_{1}\left(a_{1}(x)+a_{2}(x)\right)+c_{2}\left(b_{1}(x)+b_{2}(x)\right)+ \\
\left.+(B g)(x)+B^{2} g\right)(x) .
\end{gathered}
$$

From (5) and (8) it follows

$$
\begin{gathered}
u(x)=\left(B^{3} u\right)(x)+c_{1}\left(x+a_{1}(x)+a_{2}(x)\right)+c_{2}\left(1+b_{1}(x)+\right. \\
\left.+b_{2}(x)\right)+g(x)+(B g)(x)+\left(B^{2} g\right)(x) .
\end{gathered}
$$

Continuing this procedure $n$ times we obtain the following integral representation for solutions of equation (1):

$$
u(x)=\left(B^{n} u\right)(x)+c_{1}\left(x+\sum_{k=1}^{n-1} a_{k}(x)\right)+c_{2}\left(1+\sum_{k=1}^{n-1} b_{k}(x)\right)+\sum_{k=0}^{n-1}\left(B^{k} g\right)(x)
$$

where $\left(B^{0} g\right)(x)=g(x)$.

Let $u(x) \in C\left[0, x_{1}\right]$. The following inequalities are easily obtained:

$$
\left|\left(B^{n} u\right)(x)\right| \leq 2|u|_{1} \cdot \frac{|a|_{0}^{n} \cdot x_{1}^{2 n}}{2^{n}}, \quad(n=1,2, \ldots),
$$




$$
\left|a_{k}(x)\right|<\frac{|a|_{0}^{k} \cdot x_{1}^{2 k}}{2^{k}} \cdot x, \quad\left|b_{k}(x)\right|<\frac{2 \cdot|a|_{0}^{k} \cdot x_{1}^{2 k}}{2^{k}},(k=1,2, \ldots),
$$

where $|f|_{1}=\max _{x \in\left[0, x_{1}\right]}|f(x)|$.

Passing to the limit with $n \rightarrow \infty$ in the representation (9) and taking inequalities (10), (3) into account we get

$$
u(x)=c_{1} I_{1}(x)+c_{2} I_{2}(x)+F(x)
$$

where

$$
I_{1}(x)=x+\sum_{k=1}^{\infty} a_{k}(x), \quad I_{2}(x)=1+\sum_{k=1}^{\infty} b_{k}(x), \quad F(x)=\sum_{k=0}^{\infty}\left(B^{k} g\right)(x) .
$$

Using the inequalities (10), (11), we receive

$$
\begin{aligned}
\left|I_{1}(x)\right| \leq & \frac{2 x}{2-|a|_{0} \cdot x_{1}^{2}}, \quad\left|I_{2}(x)\right|<\frac{2+|a|_{0} \cdot x_{1}^{2}}{2-|a|_{0} \cdot x_{1}^{2}}, \\
& |F(x)| \leq|g|_{1} \frac{2+|a|_{0} \cdot x_{1}^{2}}{2-|a|_{0} \cdot x_{1}^{2}} .
\end{aligned}
$$

From the form of functions $I_{1}(x), I_{2}(x)$ and $F(x)$ for $x \in\left[0, x_{1}\right]$, where $x_{1}$ satisfies inequality (3), it follows

$$
\begin{gathered}
I_{1}^{\prime}(x)=1+\int_{x}^{x_{1}} a(t) I_{1}(t) d t, \quad I_{2}^{\prime}(x)=\int_{x}^{x_{1}} a(t) I_{2}(t) d t, \\
F^{\prime}(x)=-\int_{x}^{x_{1}} f(t) d t+\int_{x}^{x_{1}} a(t) F(t) d t \\
I_{1}^{\prime \prime}(x)=-a(x) I_{1}(x), \quad I_{2}^{\prime \prime}(x)=-a(x) I_{2}(x), \\
F^{\prime \prime}(x)=f(x)-a(x) F(x) .
\end{gathered}
$$

Considering (14) and the form of functions $I_{1}(x), I_{2}(x), F(x)$ we get

$$
I_{1}(0)=F(0)=F^{\prime}\left(x_{1}\right)=I_{2}^{\prime}\left(x_{1}\right)=0, \quad I_{2}(0)=I_{1}^{\prime}\left(x_{1}\right)=1 .
$$

From (15) and (16) it follows, that functions $I_{1}(x), I_{2}(x)$ are particular solutions from class (2) of homogeneous equations

$$
\frac{d^{2} u}{d x^{2}}+a(x) u=0
$$

and the function $F(x)$ is solution of non-homogeneous equation (1). 
From (15) and (17) we see that the Wronskian $W(x)$ of the system of functions $I_{1}(x), I_{2}(x)$ is equal to $-I_{2}\left(x_{1}\right)$. Therefore if $I_{2}\left(x_{1}\right) \neq 0$ then the functions $I_{1}(x)$ and $I_{2}(x)$ are linear independent on $\left[0, x_{1}\right]$ and the general solution to equation (1) is determined by the formula (12).

Hence, we proved the following theorem.

Theorem. If $I_{2}\left(x_{1}\right) \neq 0$ then the function $u(x)$, given by the formula (12), is a general solution of equation (1) from class (2).

\section{Solution of boundary value problem}

For equation (1) we consider the problem D. To solve the problem D we use the solution of equation (1), given by the formula (12). Substituting the function $u(x)$, given by formula (12), into boundary conditions (4) and taking into account (17) we have

$$
c_{2}=\alpha, \quad c_{1}=\beta \text {. }
$$

Hence, the solution of problem D has a form

$$
u(x)=\beta I_{1}(x)+\alpha I_{2}(x)+F(x) .
$$

Therefore, the following theorem is proved.

Theorem. If $I_{2}\left(x_{1}\right) \neq 0$ then the problem $D$ has solution, which is given by the formula (18).

Remark. Obviously, that the results of present work and take place for $a(x), f(x) \in C\left[0, x_{1}\right]$. In this case the solutions given by the formulas (12) and (18) belong to the class $C^{2}\left[0, x_{1}\right]$.

\section{References}

[1] E. Kamke, Differentialgleichungen, Lösungsmethoden und Lösungen, Teil 1, Akad. Verlag, Leipzig, 1959.

[2] M. F. Fedoryk, The asymptotically methods for lineary ordinary differential equations, Nauka, Moskow, 1983.

[3] M. A. Naimark, Linear Differential Operators, 1969.

[4] G. Sansone, Ordinary Differential Equations, V.1, 1953.

[5] E. A. Coddington, N. Levinson, Theory of ordinary differential equations, 1958. 
[6] A. Boucherif, B. Chanane, Boundary value problems for second order differential inclusions // Int. I. Differ. Equ. Appl. 7 (2003), no. 2, 147$151,10$.

[7] A. Boucherif, B. Chanane, Second order multivalued boundary value problem // Comm. Appl. Nonlinear Anal. 11(2004), no. 1, 85-91.

[8] T. Donchev, M. Quincampoix, A two point boundary value problem for a class of differential inclusions // I. Nonlinear Convex Anal. 5 (2004), no. 1, 59-69.

[9] L. Erbe, R. Ma, C. C. Tisdell, On two point boundary value problems for second order differential inclusions // Dynam. Systems Appl. 15 (2006), no. $1,79-88$.

[10] A. Tungatarov, D. K. Akhmed-Zaki, General solution of second order linear ordinary differential equations with variable coefficients // Journal of Inequalities and Special Functions. 2012, vol.3, no. 4, 42-49.

[11] A. Tungatarov, D. K. Akhmed-Zaki, Cauchy problem for one class of ordinary differential equations // Int.J. of Mathematical Analysis. 2012, vol.6, no. 14, 695-699.

Received: November 21, 2014; Published: January 9, 2015 